\title{
Logarithmically Improved Blow up Criterion for Smooths Solution to the 3D Micropolar Fluid Equations
}

\author{
Yin-Xia Wang ${ }^{1}$ and Hengjun Zhao ${ }^{2}$ \\ ${ }^{1}$ School of Mathematics and Information Sciences, North China University of Water Resources and \\ Electric Power, Zhengzhou 450011, China \\ ${ }^{2}$ Department of Mathematical and Physical Sciences, Henan Institute of Engineering, \\ Zhengzhou 451191, China
}

Correspondence should be addressed to Yin-Xia Wang, yinxia117@126.com

Received 8 January 2012; Accepted 24 April 2012

Academic Editor: Mina Abd-El-Malek

Copyright (C) 2012 Y.-X. Wang and H. Zhao. This is an open access article distributed under the Creative Commons Attribution License, which permits unrestricted use, distribution, and reproduction in any medium, provided the original work is properly cited.

Blow-up criteria of smooth solutions for the 3D micropolar fluid equations are investigated. Logarithmically improved blow-up criteria are established in the Morrey-Campanto space.

\section{Introduction}

This paper concerns the initial value problem for the micropolar fluid equations in $\mathbb{R}^{3}$

$$
\begin{gathered}
\partial_{t} u-(\mu+\chi) \Delta u+u \cdot \nabla u+\nabla p-\chi \nabla \times w=0, \\
\partial_{t} w-\gamma \Delta w-\kappa \nabla \nabla \cdot w+2 \chi w+u \cdot \nabla w-\chi \nabla \times u=0, \\
\nabla \cdot u=0
\end{gathered}
$$

with the initial value

$$
t=0: \quad u=u_{0}(x), \quad w=w_{0}(x)
$$

where $u(t, x), w(t, x)$, and $p(t, x)$ stand for the velocity field, microrotation field, and the scalar pressure, respectively. And $v>0$ is the Newtonian kinetic viscosity, $\kappa>0$ is the dynamics micro-rotation viscosity, and $\alpha, \beta, \gamma>0$ are the angular viscosity (see, i.e., Lukaszewicz [1]).

The micropolar fluid equations were first proposed by Eringen [2]. It is a type of fluids which exhibits the micro-rotational effects and micro-rotational inertia and can be viewed 
as a non-Newtonian fluid. Physically, it may represent adequately the fluids consisting of bar-like elements. Certain anisotropic fluids, for example, liquid crystals that are made up of dumbbell molecules, are of the same type. For more background, we refer to [1] and references therein. Besides their physical applications, the micropolar fluid equations are also mathematically significant. Fundamental mathematical issues such as the global regularity of their solutions have generated extensive research, and many interesting results have been obtained (see [3-8]). Regularity criterion of weak solutions to (1.1) and (1.2) in terms of the pressure was obtained (see [4]). Gala [5] established a Serrin-type regularity criterion for the weak solutions to (1.1) and (1.2) in Morrey-Campanato space. Wang and Chen [7] established the regularity criteria of weak solutions to (1.1) and (1.2) via the derivative of the velocity in one direction. A new logarithmically improved blow-up criterion of smooth solutions to (1.1) and (1.2) in an appropriate homogeneous Besov space is established by Wang and Yuan [8].

If $\kappa=0$ and $w=0$, then (1.1) reduces to be the Navier-Stokes equations. Besides its physical applications, the Navier-Stokes equations are also mathematically significant. In the last century, Leray [9] and Hopf [10] constructed weak solutions to the Navier-Stokes equations. The solution is called the Leray-Hopf weak solution. Later on, much effort has been devoted to establish the global existence and uniqueness of smooth solutions to the Navier-Stokes equations. Different criteria for regularity of the weak solutions have been proposed and many interesting results are established (see [11-26]). Regularity criteria of weak solutions to the Navier-Stokes equations in Morrey space were obtained in $[13,21]$.

The main aim of this paper is to establish two logarithmically blow-up criteria of smooth solution to (1.1), (1.2). Our results state as follows.

Theorem 1.1. Let $u_{0}, w_{0} \in H^{m}\left(\mathbb{R}^{3}\right)(m \geq 3)$ with $\nabla \cdot u_{0}=0$. Assume that $(u, w)$ is a smooth solution to (1.1) and (1.2) on [0, T). If $u$ satisfies

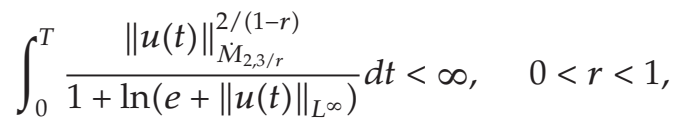

then the solution $(u, w)$ can be extended beyond $t=T$.

We have the following corollary immediately.

Corollary 1.2. Let $u_{0}, w_{0} \in H^{m}\left(\mathbb{R}^{3}\right)(m \geq 3)$ with $\nabla \cdot u_{0}=0$. Assume that $(u, w)$ is a smooth solution to (1.1) and (1.2) on [0,T). Suppose that $T$ is the maximal existence time, then

$$
\int_{0}^{T} \frac{\|u(t)\|_{\dot{M}_{2,3 / r} /(1-r)}^{2}}{1+\ln \left(e+\|u(t)\|_{L^{\infty}}\right)} d t=\infty, \quad 0<r<1 .
$$

Theorem 1.3. Let $u_{0}, w_{0} \in H^{m}\left(\mathbb{R}^{3}\right)(m \geq 3)$ with $\nabla \cdot u_{0}=0$. Assume that $(u, w)$ is a smooth solution to (1.1) and (1.2) on [0, T). If u satisfies

$$
\int_{0}^{T} \frac{\|\nabla u(t)\|_{\dot{M}_{2,3 / r}}^{2 /(2-r)}}{1+\ln \left(e+\|\nabla u(t)\|_{L^{\infty}}\right)} d t<\infty, \quad 0<r<1,
$$

then the solution $(u, w)$ can be extended beyond $t=T$. 
One has the following corollary immediately.

Corollary 1.4. Let $u_{0}, w_{0} \in H^{m}\left(\mathbb{R}^{3}\right)(m \geq 3)$ with $\nabla \cdot u_{0}=0$. Assume that $(u, w)$ is a smooth solution to (1.1) and (1.2) on [0,T). Suppose that $T$ is the maximal existence time, then

$$
\int_{0}^{T} \frac{\|\nabla u(t)\|_{\dot{M}_{2,3 / r}}^{2 /(2-r)}}{1+\ln \left(e+\|\nabla u(t)\|_{L^{\infty}}\right)} d t=\infty, \quad 0<r<1
$$

The paper is organized as follows. We first state some important inequalities in Section 2 , which play an important role in the proof of our main result. Then, we prove the main result in Section 3 and Section 4, respectively.

\section{Preliminaries}

Firstly, we recall the definition and some properties of the space that we are going to use. The space plays an important role in studying the regularity of solutions to nonlinear differential equations.

Definition 2.1. For $1<p \leq q \leq+\infty$, the Morrey-Campanato space $\dot{M}_{p, q}$ is defined by

$$
\dot{M}_{p, q}=\left\{f \in L_{\mathrm{loc}}^{p}\left(\mathbb{R}^{3}\right) \mid\|f\|_{\dot{M}_{p, q}}=\sup _{x \in \mathbb{R}^{3} R>0} \sup _{R>0} R^{3 / q-3 / p}\|f\|_{L^{p}(B(x, R))}<\infty\right\},
$$

where $B(x, R)$ denotes the ball of center $x$ with radius $R$.

It is easy to verify that $\dot{M}_{p, q}$ is a Banach space under the norm $\|\cdot\|_{\dot{M}_{p, q}}$. Furthermore, it is easy to check the following:

$$
\|f(\lambda \cdot)\|_{\dot{M}_{p, q}}=\lambda^{-3 / q}\|f\|_{\dot{M}_{p, q}}, \quad \lambda>0 .
$$

Morrey-Campanato spaces can be seen as a complement to $L^{p}$ spaces. In fact, for $p \leq q$, one has

$$
L^{q}=\dot{M}_{q, q} \subset \dot{M}_{p, q}
$$

one has the following comparison between Lorentz spaces and Morrey-Campanato spaces: for $p \geq 2$,

$$
L^{3 / r}\left(\mathbb{R}^{3}\right) \subset L^{3 / r, \infty}\left(\mathbb{R}^{3}\right) \subset \dot{M}_{p, 3 / r}\left(\mathbb{R}^{3}\right),
$$

where $L^{p, \infty}$ denotes the usual Lorentz (weak $L^{p}$ ) space.

In the proof of our main result, we need the following lemma which was given in [27]. 
Lemma 2.2. For $0 \leq r<3 / 2$, the space $\dot{Z}_{r}$ is defined as the space of $f(x) \in L_{\mathrm{loc}}^{2}\left(\mathbb{R}^{3}\right)$ such that

$$
\|f\|_{\dot{Z}_{r}}=\sup _{\|g\|_{B_{2,1}^{r}} \leq 1}\|f g\|_{L^{2}}<\infty
$$

Then $f \in \dot{M}_{2,3 / r}$ if and only if $f \in \dot{Z}_{r}$ with equivalence of norms. And the fact that

$$
L^{2} \bigcap \dot{H}^{1} \subset \dot{B}_{2,1}^{r} \subset \dot{H}^{r}, \quad 0<r<1,
$$

one has

$$
\dot{X}_{r} \subset \dot{M}_{2,3 / r}
$$

where $\dot{X}_{r}$ denotes the pointwise multiplier space from $\dot{H}^{r}$ to $L^{2}$.

We need the following lemma that is basically established in [28]. For completeness, the proof will be also sketched here.

Lemma 2.3. For $0<r<1$, the inequality

$$
\|f\|_{\dot{B}_{2,1}^{r}} \leq C\|f\|_{L^{2}}^{1-r}\|\nabla f\|_{L^{2}}^{r}
$$

holds, where $C$ is a positive constant that depends on $r$.

Proof. It follows from the definition of Besov spaces that

$$
\begin{aligned}
\|f\|_{\dot{B}_{2,1}^{r}} & =\sum_{i \in \mathbb{Z}} 2^{i r}\left\|\Delta_{i} f\right\|_{L^{2}} \\
& \leq \sum_{i \leq j} 2^{i r}\left\|\Delta_{i} f\right\|_{L^{2}}+\sum_{i>j} 2^{i(r-1)} 2^{i}\left\|\Delta_{i} f\right\|_{L^{2}} \\
& \leq\left(\sum_{i \leq j} 2^{2 i r}\right)^{1 / 2}\left(\sum_{i \leq j}\left\|\Delta_{i} f\right\|_{L^{2}}^{2}\right)^{1 / 2}+\left(\sum_{i \leq j} 2^{2 i(r-1)}\right)^{1 / 2}\left(\sum_{i>j} 2^{2 i}\left\|\Delta_{i} f\right\|_{L^{2}}^{2}\right)^{1 / 2} \\
& \leq C\left(2^{j r}\|f\|_{L^{2}}+2^{j(r-1)}\|f\|_{H^{1}}\right) \\
& =C\left(2^{j r} A^{-r}+2^{j(r-1)} A^{1-r}\right)\|f\|_{L^{2}}^{1-r}\|f\|_{\dot{H}^{1 \prime}}^{r}
\end{aligned}
$$

where $A=\left(\|f\|_{H^{1}}\right) /\left(\|f\|_{L^{2}}\right)$. Choosing $j$ such that $1 / 2 \leq 2^{j r} A^{-r} \leq 1$, from (2.9) we get

$$
\begin{aligned}
\|f\|_{B_{2,1}^{r}} & \leq\left(1+2^{j(r-1)} A^{1-r}\right)\|f\|_{L^{2}}^{1-r}\|f\|_{H^{1}}^{r} \\
& \leq C\left(1+\left(\frac{1}{2}\right)^{-1 / r}\right)\|f\|_{L^{2}}^{1-r}\|\nabla f\|_{L^{2}}^{r} .
\end{aligned}
$$

Therefore, we have completed the proof of Lemma 2.3. 
Journal of Applied Mathematics

The following Lemma comes from [29].

Lemma 2.4. Assume that $1<p<\infty$. For $f, g \in W^{m, p}$, and $1<q \leq \infty, 1<r<\infty$, one has

$$
\left\|\nabla^{\alpha}(f g)-f \nabla^{\alpha} g\right\|_{L^{p}} \leq C\left(\|\nabla f\|_{L^{q_{1}}}\left\|\nabla^{\alpha-1} g\right\|_{L^{r_{1}}}+\|g\|_{L^{q_{2}}}\left\|\nabla^{\alpha} f\right\|_{L^{r_{2}}}\right)
$$

where $1 \leq \alpha \leq m$ and $1 / p=1 / q_{1}+1 / r_{1}=1 / q_{2}+1 / r_{2}$.

In order to prove Theorem 1.1, we need the following interpolation inequalities in three space dimensions.

Lemma 2.5. In three space dimensions, the following inequalities

$$
\begin{gathered}
\|\nabla f\|_{L^{4}} \leq C\|f\|_{L^{2}}^{1 / 8}\left\|\nabla^{2} f\right\|_{L^{2}}^{7 / 8} \\
\|f\|_{L^{4}} \leq C\|f\|_{L^{2}}^{3 / 4}\left\|\nabla^{3} f\right\|_{L^{2}}^{1 / 4} \\
\left\|\nabla^{2} f\right\|_{L^{4}} \leq C\|f\|_{L^{2}}^{1 / 12}\left\|\nabla^{3} f\right\|_{L^{2}}^{11 / 12} \\
\left\|\nabla^{2} f\right\|_{L^{2}} \leq C\|f\|_{L^{2}}^{1 / 3}\left\|\nabla^{3} f\right\|_{L^{2}}^{2 / 3}
\end{gathered}
$$

hold.

\section{Proof of Theorem 1.1}

Proof. Multiplying the first equation of (1.1) by $u$ and integrating with respect to $x$ over $\mathbb{R}^{3}$, using integration by parts, we obtain

$$
\frac{1}{2} \frac{d}{d t}\|u(t)\|_{L^{2}}^{2}+(\mu+x)\|\nabla u(t)\|_{L^{2}}^{2}=x \int_{\mathbb{R}^{3}}(\nabla \times w) \cdot u d x
$$

Similarly, we get

$$
\frac{1}{2} \frac{d}{d t}\|w(t)\|_{L^{2}}^{2}+\gamma\|\nabla w(t)\|_{L^{2}}^{2}+\kappa\|\nabla \cdot w\|_{L^{2}}^{2}+2 x\|w\|_{L^{2}}^{2}=x \int_{\mathbb{R}^{3}}(\nabla \times u) \cdot w d x
$$

Summing up (3.1) and (3.2), we deduce thats

$$
\begin{aligned}
\frac{1}{2} \frac{d}{d t}\left(\|u(t)\|_{L^{2}}^{2}+\|w(t)\|_{L^{2}}^{2}\right)+(\mu+x)\|\nabla u(t)\|_{L^{2}}^{2} \\
+\gamma\|\nabla w(t)\|_{L^{2}}^{2}+\kappa\|\nabla \cdot w\|_{L^{2}}^{2}+2 x\|w\|_{L^{2}}^{2} \\
=x \int_{\mathbb{R}^{3}}(\nabla \times w) \cdot u d x+x \int_{\mathbb{R}^{3}}(\nabla \times u) \cdot w d x
\end{aligned}
$$


Using integration by parts and Cauchy's inequality, we obtain

$$
x \int_{\mathbb{R}^{3}}(\nabla \times w) \cdot u d x+x \int_{\mathbb{R}^{3}}(\nabla \times u) \cdot w d x \leq x\|\nabla u\|_{L^{2}}^{2}+x\|w\|_{L^{2}}^{2}
$$

Combining (3.3) and (3.4) yields

$$
\frac{1}{2} \frac{d}{d t}\left(\|u(t)\|_{L^{2}}^{2}+\|w(t)\|_{L^{2}}^{2}\right)+\mu\|\nabla u(t)\|_{L^{2}}^{2}+\gamma\|\nabla w(t)\|_{L^{2}}^{2}+\kappa\|\nabla \cdot w\|_{L^{2}}^{2}+x\|w\|_{L^{2}}^{2} \leq 0 .
$$

Integrating with respect to $t$, we have

$$
\begin{aligned}
\|u(t)\|_{L^{2}}^{2} & +\|w(t)\|_{L^{2}}^{2}+2 \int_{0}^{t}\left(\mu\|\nabla u(\tau)\|_{L^{2}}^{2}+\gamma\|\nabla w(\tau)\|_{L^{2}}^{2}\right) d \tau \\
& +2 \kappa \int_{0}^{t}\|\nabla \cdot w(\tau)\|_{L^{2}}^{2} d \tau+2 \chi \int_{0}^{t}\|w(\tau)\|_{L^{2}}^{2} d \tau \leq\left\|u_{0}\right\|_{L^{2}}^{2}+\left\|w_{0}\right\|_{L^{2}}^{2}
\end{aligned}
$$

Taking $\nabla$ to the first equation of (1.1), then multiplying the resulting equation by $\nabla u$ and using integration by parts, we obtain

$$
\frac{1}{2} \frac{d}{d t}\|\nabla u(t)\|_{L^{2}}^{2}+(\mu+x)\left\|\nabla^{2} u(t)\right\|_{L^{2}}^{2}=-\int_{\mathbb{R}^{3}} \nabla(u \cdot \nabla u) \nabla u d x+x \int_{\mathbb{R}^{3}} \nabla(\nabla \times w) \nabla u d x
$$

Similarly, we get

$$
\begin{array}{r}
\frac{1}{2} \frac{d}{d t}\|\nabla w(t)\|_{L^{2}}^{2}+\gamma\left\|\nabla^{2} w(t)\right\|_{L^{2}}^{2}+\kappa\|\nabla \cdot \nabla w\|_{L^{2}}^{2}+2 \chi\|\nabla w\|_{L^{2}}^{2} \\
=-\int_{\mathbb{R}^{3}} \nabla(u \cdot \nabla w) \cdot \nabla w d x+x \int_{\mathbb{R}^{3}} \nabla(\nabla \times u) \cdot \nabla w d x .
\end{array}
$$

Combining (3.7) and (3.8) yields

$$
\begin{aligned}
\frac{1}{2} \frac{d}{d t}( & \left.\|\nabla u(t)\|_{L^{2}}^{2}+\|\nabla w(t)\|_{L^{2}}^{2}\right)+(\mu+x)\left\|\nabla^{2} u(t)\right\|_{L^{2}}^{2} \\
& +\gamma\left\|\nabla^{2} w(t)\right\|_{L^{2}}^{2}+\kappa\|\nabla \nabla \cdot w\|_{L^{2}}^{2}+2 \chi\|\nabla w\|_{L^{2}}^{2} \\
= & -\int_{\mathbb{R}^{3}} \nabla(u \cdot \nabla u) \nabla u d x+\chi \int_{\mathbb{R}^{3}} \nabla(\nabla \times w) \nabla u d x \\
& -\int_{\mathbb{R}^{3}} \nabla(u \cdot \nabla w) \nabla w d x+\chi \int_{\mathbb{R}^{3}} \nabla(\nabla \times u) \nabla w d x .
\end{aligned}
$$

Using integration by parts and Cauchy's inequality, we obtain

$$
x \int_{\mathbb{R}^{3}} \nabla(\nabla \times w) \cdot \nabla u d x+x \int_{\mathbb{R}^{3}} \nabla(\nabla \times u) \cdot \nabla w d x \leq x\left\|\nabla^{2} u\right\|_{L^{2}}^{2}+x\|\nabla w\|_{L^{2}}^{2} .
$$


Using Hölder's inequality, (2.8), and Young's inequality, we obtain

$$
\begin{aligned}
-\int_{\mathbb{R}^{3}} & \nabla(u \cdot \nabla u) \nabla u d x \\
\leq & \|\nabla u\|_{L^{2}}\|\nabla u \nabla u\|_{L^{2}} \\
\leq & C\|\nabla u\|_{\dot{M}_{2,3 / r}}\|\nabla u\|_{\dot{B}_{2,1}^{r}}\|\nabla u\|_{L^{2}} \\
\leq & C\|\nabla u\|_{\dot{M}_{2,3 / r}}\|\nabla u\|_{\dot{B}_{2,1}^{r}}^{2-r}\left\|\nabla^{2} u\right\|_{L^{2}}^{r} \\
\leq & \frac{\mu}{2}\left\|\nabla^{2} u(t)\right\|_{L^{2}}^{2}+C\|\nabla u\|_{M_{2,3 / r} /(2-r)}^{2}\|\nabla u\|_{L^{2}}^{2} .
\end{aligned}
$$

Similarly, we have the following estimate:

$$
\begin{aligned}
-\int_{\mathbb{R}^{3}} & \nabla(u \cdot \nabla w) \nabla w d x \\
\leq & \leq \nabla w\left\|_{L^{2}}\right\| \nabla u \nabla w \|_{L^{2}} \\
\leq & C\|\nabla u\|_{\dot{M}_{2,3 / r}}\|\nabla w\|_{\dot{B}_{2,1}^{r}}^{r}\|\nabla w\|_{L^{2}} \\
\leq & C\|\nabla u\|_{\dot{M}_{2,3 / r}}\|\nabla w\|_{\dot{B}_{2,1}^{r}}^{2-r}\left\|\nabla^{2} w\right\|_{L^{2}}^{r} \\
\leq & \frac{\gamma}{2}\left\|\nabla^{2} w(t)\right\|_{L^{2}}^{2}+C\|\nabla u\|_{\dot{M}_{2,3 / r}}^{2 /(2-r)}\|\nabla w\|_{L^{2}}^{2} .
\end{aligned}
$$

Combining (3.9)-(3.12) yields

$$
\begin{aligned}
& \frac{d}{d t}\left(\|\nabla u(t)\|_{L^{2}}^{2}+\|\nabla w(t)\|_{L^{2}}^{2}\right)+\mu\left\|\nabla^{2} u(t)\right\|_{L^{2}}^{2}+\gamma\left\|\nabla^{2} w\right\|_{L^{2}}^{2}+\kappa\|\nabla \nabla \cdot w\|_{L^{2}}^{2}+x\|\nabla w\|_{L^{2}}^{2} \\
& \quad \leq C\|\nabla u\|_{M_{2,3 / r} /(2-r)}^{2}\left(\|\nabla u\|_{L^{2}}^{2}+\|\nabla w\|_{L^{2}}^{2}\right) \\
& \quad \leq C \frac{\|\nabla u\|_{M_{2,3 / r}}^{2 /(2-r)}}{1+\ln \left(e+\|\nabla u\|_{L^{\infty}}\right)}\left(\|\nabla u\|_{L^{2}}^{2}+\|\nabla w\|_{L^{2}}^{2}\right)\left(1+\ln \left(e+\|\nabla u\|_{L^{\infty}}\right)\right) \\
& \quad \leq C \frac{\|\nabla u\|_{\dot{M}_{2,3 / r}}^{2 /(2-r)}}{1+\ln \left(e+\|\nabla u\|_{L^{\infty}}\right)}\left(\|\nabla u\|_{L^{2}}^{2}+\|\nabla w\|_{L^{2}}^{2}\right)\left(1+\ln \left(e+\left\|\nabla^{3} u\right\|_{L^{2}}+\left\|\nabla^{3} w\right\|_{L^{2}}\right)\right),
\end{aligned}
$$

where we have used

$$
H^{2}\left(\mathbb{R}^{3}\right) \hookrightarrow L^{\infty}\left(\mathbb{R}^{3}\right)
$$

For any $T_{0} \leq t \leq T$, we set

$$
\vartheta(t)=\sup _{T_{0} \leq \tau \leq t}\left(\left\|\nabla^{3} u(\tau)\right\|_{L^{2}}^{2}+\left\|\nabla^{3} w(\tau)\right\|_{L^{2}}^{2}\right)
$$


Thus, from (3.13), we have

$$
\begin{gathered}
\frac{d}{d t}\left(\|\nabla u(t)\|_{L^{2}}^{2}+\|\nabla w(t)\|_{L^{2}}^{2}\right)+\mu\left\|\nabla^{2} u(t)\right\|_{L^{2}}^{2}+\gamma\left\|\nabla^{2} w\right\|_{L^{2}}^{2}+\kappa\|\nabla \nabla \cdot w\|_{L^{2}}^{2}+x\|\nabla w\|_{L^{2}}^{2} \\
\leq C \frac{\|\nabla u\|_{M_{2,3 / r}}^{2 /(2-r)}}{1+\ln \left(e+\|\nabla u\|_{L^{\infty}}\right)}\left(\|\nabla u\|_{L^{2}}^{2}+\|\nabla w\|_{L^{2}}^{2}\right)(1+\ln (e+\vartheta(t))), \quad \forall T_{0} \leq t<T .
\end{gathered}
$$

It follows from (3.8) and Gronwall's inequality that

$$
\begin{aligned}
\| \nabla u(t) & \left\|_{L^{2}}^{2}+\right\| \nabla w(t) \|_{L^{2}}^{2} \\
& \leq\left(\left\|\nabla u\left(T_{0}\right)\right\|_{L^{2}}^{2}+\left\|\nabla w\left(T_{0}\right)\right\|_{L^{2}}^{2}\right) \exp \left\{C(1+\ln (e+\vartheta(t))) \int_{T_{0}}^{t} \frac{\|\nabla u(\tau)\|_{\dot{M}_{2,3 / r}}^{2 /(2-r)}}{1+\ln \left(e+\|\nabla u\|_{L^{\infty}}\right)} d \tau\right\} \\
& \leq C_{0} \exp \{C \varepsilon[1+\ln (e+\vartheta(t))]\} \\
& \leq C_{0} \exp \{2 C \varepsilon[\ln (e+\vartheta(t))]\} \\
& \leq C_{0}(e+\vartheta(t))^{2 C \varepsilon},
\end{aligned}
$$

provided that

$$
\int_{T_{0}}^{t} \frac{\|\nabla u(\tau)\|_{\dot{M}_{2,3 / r}}^{2 /(2-r)}}{1+\ln \left(e+\|\nabla u\|_{L^{\infty}}\right)} d \tau<\varepsilon \ll 1
$$

where $C_{0}=\left\|\nabla u\left(T_{0}\right)\right\|_{L^{2}}^{2}+\left\|\nabla w\left(T_{0}\right)\right\|_{L^{2}}^{2}$.

Applying $\nabla^{m}$ to the first equation of (1.1), then multiplying the resulting equation by $\nabla^{m} u$ and using integration by parts, we have

$\frac{1}{2} \frac{d}{d t}\left\|\nabla^{m} u(t)\right\|_{L^{2}}^{2}+(\mu+x)\left\|\nabla^{m+1} u(t)\right\|_{L^{2}}^{2}=-\int_{\mathbb{R}^{3}} \nabla^{m}(u \cdot \nabla u) \nabla^{m} u d x+x \int_{\mathbb{R}^{3}} \nabla^{m}(\nabla \times w) \nabla^{m} u d x$

Likewise, from the second equation of (1.1), we obtain

$$
\begin{gathered}
\frac{1}{2} \frac{d}{d t}\left\|\nabla^{m} w(t)\right\|_{L^{2}}^{2}+\gamma\left\|\nabla^{m+1} w(t)\right\|_{L^{2}}^{2}+\kappa\left\|\nabla^{m} \nabla \cdot w\right\|_{L^{2}}^{2}+2 \chi\left\|\nabla^{m} w(t)\right\|_{L^{2}}^{2} \\
=-\int_{\mathbb{R}^{3}} \nabla^{m}(u \cdot \nabla w) \nabla^{m} w d x+x \int_{\mathbb{R}^{3}} \nabla^{m}(\nabla \times u) \nabla^{m} w d x .
\end{gathered}
$$


Using $\nabla \cdot u=0$ and (3.19) and (3.20), we have

$$
\begin{aligned}
\frac{1}{2} \frac{d}{d t}( & \left.\left\|\nabla^{m} u(t)\right\|_{L^{2}}^{2}+\left\|\nabla^{m} w(t)\right\|_{L^{2}}^{2}\right)+(\mu+x)\left\|\nabla^{m+1} u(t)\right\|_{L^{2}}^{2} \\
& +\gamma\left\|\nabla^{m+1} w(t)\right\|_{L^{2}}^{2}+\kappa\left\|\nabla^{m} \nabla \cdot w\right\|_{L^{2}}^{2}+2 x\left\|\nabla^{m} w(t)\right\|_{L^{2}}^{2} \\
= & -\int_{\mathbb{R}^{3}}\left[\nabla^{m}(u \cdot \nabla u)-u \cdot \nabla \nabla^{m} u\right] \nabla^{m} u d x+x \int_{\mathbb{R}^{3}} \nabla^{m}(\nabla \times w) \nabla^{m} u d x \\
& -\int_{\mathbb{R}^{3}}\left[\nabla^{m}(u \cdot \nabla w)-u \cdot \nabla \nabla^{m} w\right] \nabla^{m} w d x+x \int_{\mathbb{R}^{3}} \nabla^{m}(\nabla \times u) \nabla^{m} w d x .
\end{aligned}
$$

In what follows, for simplicity, we will set $m=3$.

By Hölder's inequality, (2.11), (2.12), and Young's inequality, we obtain

$$
\begin{aligned}
-\int_{\mathbb{R}^{3}} & {\left[\nabla^{3}(u \cdot \nabla u)-u \cdot \nabla \nabla^{3} u\right] \nabla^{3} u d x } \\
\leq & \left\|\nabla^{3}(u \cdot \nabla u)-u \cdot \nabla \nabla^{3} u\right\|_{L^{2}}\left\|\nabla^{3} u\right\|_{L^{2}} \\
\leq & C\|\nabla u\|_{L^{4}}\left\|\nabla^{3} u\right\|_{L^{4}}\left\|\nabla^{3} u\right\|_{L^{2}} \\
\leq & C\|\nabla u\|_{L^{2}}^{3 / 4}\left\|\nabla^{4} u\right\|_{L^{2}}^{1 / 4}\|\nabla u\|_{L^{2}}^{1 / 12}\left\|\nabla^{4} u\right\|_{L^{2}}^{11 / 12}\|\nabla u\|_{L^{2}}^{1 / 3}\left\|\nabla^{4} u\right\|_{L^{2}}^{2 / 3} \\
\leq & C\|\nabla u\|_{L^{2}}^{7 / 6}\left\|\nabla^{4} u\right\|_{L^{2}}^{11 / 6} \\
\leq & \frac{\mu}{4}\left\|\nabla^{4} u\right\|_{L^{2}}^{2}+C\|\nabla u\|_{L^{2}}^{14} \\
\leq & \frac{\mu}{4}\left\|\nabla^{4} u\right\|_{L^{2}}^{2}+C(e+\vartheta(t))^{14 C \varepsilon}, \\
-\int_{\mathbb{R}^{3}} & {\left[\nabla^{3}(u \cdot \nabla w)-u \cdot \nabla \nabla^{3} w\right] \nabla^{3} w d x } \\
\leq & \left\|\nabla^{3}(u \cdot \nabla w)-u \cdot \nabla \nabla^{3} w\right\|_{L^{2}}\left\|\nabla^{3} w\right\|_{L^{2}} \\
\leq & C\|\nabla u\|_{L^{4}}\left\|\nabla^{3} w\right\|_{L^{4}}\left\|\nabla^{3} w\right\|_{L^{2}}+\|\nabla w\|_{L^{4}}\left\|\nabla^{3} u\right\|_{L^{4}}\left\|\nabla^{3} w\right\|_{L^{2}} \\
\leq & C\|\nabla u\|_{L^{2}}^{3 / 4}\left\|\nabla^{4} u\right\|_{L^{2}}^{1 / 4}\|\nabla w\|_{L^{2}}^{1 / 12}\left\|\nabla^{4} w\right\|_{L^{2}}^{11 / 12}\|\nabla w\|_{L^{2}}^{1 / 3}\left\|\nabla^{4} w\right\|_{L^{2}}^{2 / 3} \\
& +C\|\nabla w\|_{L^{2}}^{3 / 4}\left\|\nabla^{4} w\right\|_{L^{2}}^{1 / 4}\|\nabla u\|_{L^{2}}^{1 / 12}\left\|\nabla^{4} u\right\|_{L^{2}}^{11 / 12}\|\nabla w\|_{L^{2}}^{1 / 3}\left\|\nabla^{4} w\right\|_{L^{2}}^{2 / 3} \\
\leq & \frac{\mu}{4}\left\|\nabla^{4} u\right\|_{L^{2}}^{2}+\frac{\gamma}{2}\left\|\nabla^{4} w\right\|_{L^{2}}^{2}+C\|\nabla u\|_{L^{2}}^{9}\|\nabla w\|_{L^{2}}^{5}+C\|\nabla u\|_{L^{2}}\|\nabla w\|_{L^{2}}^{13} \\
\leq & \frac{\mu}{4}\left\|\nabla^{4} u\right\|_{L^{2}}^{2}+\frac{\gamma}{2}\left\|\nabla^{4} w\right\|_{L^{2}}^{2}+C(e+v(t))^{14 C \varepsilon} \cdot
\end{aligned}
$$

It follows from integration by parts and Cauchy's inequality that

$$
x \int_{\mathbb{R}^{3}} \nabla^{3}(\nabla \times w) \nabla^{3} u d x+x \int_{\mathbb{R}^{3}} \nabla^{3}(\nabla \times u) \nabla^{3} w d x \leq x\left\|\nabla^{4} u(t)\right\|_{L^{2}}^{2}+x\left\|\nabla^{3} w(t)\right\|_{L^{2}}^{2} .
$$


Combining (3.21)-(3.24) yields

$$
\begin{aligned}
& \frac{1}{2} \frac{d}{d t}\left(\left\|\nabla^{m} u(t)\right\|_{L^{2}}^{2}+\left\|\nabla^{m} w(t)\right\|_{L^{2}}^{2}\right)+(\mu+\chi)\left\|\nabla^{m+1} u(t)\right\|_{L^{2}}^{2} \\
& +\gamma\left\|\nabla^{m+1} w(t)\right\|_{L^{2}}^{2}+\kappa\left\|\nabla^{m} \nabla \cdot w\right\|_{L^{2}}^{2}+2 \chi\left\|\nabla^{m} w(t)\right\|_{L^{2}}^{2} \\
& \leq C(e+\vartheta(t))^{14 C \varepsilon}, \quad \forall T_{0} \leq t<T \text {. }
\end{aligned}
$$

Taking $\varepsilon$ small enough yields

$$
\frac{d}{d t}\left(\left\|\nabla^{3} u\right\|_{L^{2}}^{2}+\left\|\nabla^{3} w\right\|_{L^{2}}^{2}\right) \leq C(e+\vartheta(t)), \quad T_{0} \leq t<T
$$

for all $T_{0} \leq t<T$.

Integrating (3.26) with respect to time from $T_{0}$ to $\tau$, we have

$$
e+\left\|\nabla^{3} u(\tau)\right\|_{L^{2}}^{2}+\left\|\nabla^{3} w(\tau)\right\|_{L^{2}}^{2} \leq e+\left\|\nabla^{3} u\left(T_{0}\right)\right\|_{L^{2}}^{2}+\left\|\nabla^{3} w\left(T_{0}\right)\right\|_{L^{2}}^{2}+C_{2} \int_{T_{0}}^{\tau}(e+\vartheta(s)) d s
$$

Owing to (3.27), we get

$$
e+\vartheta(t) \leq e+\left\|\nabla^{3} u\left(T_{0}\right)\right\|_{L^{2}}^{2}+\left\|\nabla^{3} w\left(T_{0}\right)\right\|_{L^{2}}^{2}+C_{2} \int_{T_{0}}^{t}(e+\vartheta(\tau)) d \tau
$$

For all $T_{0} \leq t<T$, with help of Gronwall inequality and (3.28), we have

$$
e+\left\|\nabla^{3} u(t)\right\|_{L^{2}}^{2}+\left\|\nabla^{3} w(t)\right\|_{L^{2}}^{2} \leq C
$$

where $C$ depends on $\left\|\nabla u\left(T_{0}\right)\right\|_{L^{2}}^{2}+\left\|\nabla w\left(T_{0}\right)\right\|_{L^{2}}^{2}$. From (3.29) and (3.5), we know that $(u, w) \in$ $L^{\infty}\left(0, T ; H^{3}\left(\mathbb{R}^{3}\right)\right)$. Thus, $(u, w)$ can be extended smoothly beyond $t=T$. We have completed the proof of Theorem 1.1. 
Journal of Applied Mathematics

\section{Proof of Theorem 1.3}

We start to estimate every term on the right of (3.9). Using integration by parts, Hölder inequality, (2.8) and Young inequality, we obtain

$$
\begin{aligned}
-\int_{\mathbb{R}^{3}} & \nabla(u \cdot \nabla u) \nabla u d x \\
\leq & \left\|\nabla^{2} u\right\|_{L^{2}}\|u \nabla u\|_{L^{2}} \\
\leq & C\|u\|_{\dot{M}_{2,3 / r}}\|\nabla u\|_{\dot{B}_{2,1}^{r}}\left\|\nabla^{2} u\right\|_{L^{2}} \\
\leq & C\|u\|_{\dot{M}_{2,3 / r}}\|\nabla u\|_{\dot{B}_{2,1}^{r}}^{1-r}\left\|\nabla^{2} u\right\|_{L^{2}}^{1+r} \\
\leq & \frac{\mu}{2}\left\|\nabla^{2} u(t)\right\|_{L^{2}}^{2}+C\|u\|_{\dot{M}_{2,3 / r}}^{2 /(1-r)}\|\nabla u\|_{L^{2}}^{2} .
\end{aligned}
$$

Similarly, we have the following estimate

$$
\begin{aligned}
-\int_{\mathbb{R}^{3}} & \nabla(u \cdot \nabla w) \nabla w d x \\
\leq & \left\|\nabla^{2} w\right\|_{L^{2}}\|u \nabla w\|_{L^{2}} \\
\leq & C\|u\|_{\dot{M}_{2,3 / r}}\|\nabla w\|_{\dot{B}_{2,1}^{r}}\left\|\nabla^{2} w\right\|_{L^{2}} \\
\leq & C\|u\|_{\dot{M}_{2,3 / r}}\|\nabla w\|_{\dot{B}_{2,1}^{r}}^{1-r}\left\|\nabla^{2} w\right\|_{L^{2}}^{1+r} \\
\leq & \frac{\gamma}{2}\left\|\nabla^{2} w(t)\right\|_{L^{2}}^{2}+C\|u\|_{\dot{M}_{2,3 / r}}^{2 /(1-r)}\|\nabla w\|_{L^{2}}^{2} .
\end{aligned}
$$

Thus from (3.9), (3.10), (4.1), and (4.2), we obtain

$$
\begin{gathered}
\frac{d}{d t}\left(\|\nabla u(t)\|_{L^{2}}^{2}+\|\nabla w(t)\|_{L^{2}}^{2}\right)+\mu\left\|\nabla^{2} u(t)\right\|_{L^{2}}^{2}+r\left\|\nabla^{2} w\right\|_{L^{2}}^{2}+\kappa\|\nabla \nabla \cdot w\|_{L^{2}}^{2}+x\|\nabla w\|_{L^{2}}^{2} \\
\leq C \frac{\|u\|_{M_{2,3 / r} /(1-r)}}{1+\ln \left(e+\|u\|_{L^{\infty}}\right)}\left(\|\nabla u\|_{L^{2}}^{2}+\|\nabla w\|_{L^{2}}^{2}\right)(1+\ln (e+\vartheta(t))), \quad \forall T_{0} \leq t<T .
\end{gathered}
$$

It follows from (4.3) and Gronwall's inequality that

$$
\begin{aligned}
& \|\nabla u(t)\|_{L^{2}}^{2}+\|\nabla w(t)\|_{L^{2}}^{2} \\
& \leq\left(\left\|\nabla u\left(T_{0}\right)\right\|_{L^{2}}^{2}+\left\|\nabla w\left(T_{0}\right)\right\|_{L^{2}}^{2}\right) \exp \left\{C(1+\ln (e+\vartheta(t))) \int_{T_{0}}^{t} \frac{\|u(\tau)\|_{\dot{M}_{2,3 / r}}^{2 /(1-r)}}{1+\ln \left(e+\|u\|_{L^{\infty}}\right)} d \tau\right\} \\
& \leq C_{0} \exp \{C \varepsilon[1+\ln (e+\vartheta(t))]\} \\
& \leq C_{0} \exp \{2 C \varepsilon[\ln (e+\vartheta(t))]\} \\
& \leq C_{0}(e+\vartheta(t))^{2 C \varepsilon} \text {, }
\end{aligned}
$$


provided that

$$
\int_{T_{0}}^{t} \frac{\|u(\tau)\|_{M_{2,3 / r}}^{2 /(2-r)}}{1+\ln \left(e+\|u\|_{L^{\infty}}\right)} d \tau<\varepsilon \ll 1,
$$

where $C_{0}=\left\|\nabla u\left(T_{0}\right)\right\|_{L^{2}}^{2}+\left\|\nabla w\left(T_{0}\right)\right\|_{L^{2}}^{2}$.

From (4.4), $H^{m}$ estimate for Theorem 1.3 is same as that for Theorem 1.1. Thus, Theorem 1.3 is proved.

\section{Acknowledgments}

This work was supported in part by the NNSF of China (Grant no. 11101144) and Research Initiation Project for High-level Talents (201031) of North China University of Water Resources and Electric Power.

\section{References}

[1] G. Lukaszewicz, Micropolar Fluids, Theory and Applications, Modeling and Simulation in Science, Engineering and Technology, Birkhäuser, Boston, Mass, USA, 1999.

[2] A. C. Eringen, "Theory of micropolar fluids," Journal of Mathematics and Mechanics, vol. 16, pp. 1-18, 1966.

[3] B.-Q. Dong and Z.-M. Chen, "Regularity criteria of weak solutions to the three-dimensional micropolar flows," Journal of Mathematical Physics, vol. 50, no. 10, pp. 103525-1-103525-13, 2009.

[4] B.-Q. Dong, Y. Jia, and Z.-M. Chen, "Pressure regularity criteria of the three-dimensional micropolar fluid flows," Mathematical Methods in the Applied Sciences, vol. 34, no. 5, pp. 595-606, 2011.

[5] S. Gala, "On regularity criteria for the three-dimensional micropolar fluid equations in the critical Morrey-Campanato space," Nonlinear Analysis, vol. 12, no. 4, pp. 2142-2150, 2011.

[6] E. Ortega-Torres and M. Rojas-Medar, "On the regularity for solutions of the micropolar fluid equations," Rendiconti del Seminario Matematico della Università di Padova, vol. 122, pp. 27-37, 2009.

[7] Y.-Z. Wang and Z. Chen, "Regularity criterion for weak solution to the 3D micropolar fluid equations," Journal of Applied Mathematics, vol. 2011, Article ID 456547, 12 pages, 2011.

[8] Y.-Z. Wang and H. Yuan, "A logarithmically improved blow-up criterion for smooth solutions to the 3D micropolar fluid equations," Nonlinear Analysis, vol. 13, no. 4, pp. 1904-1912, 2012.

[9] J. Leray, "Sur le mouvement d'un liquide visqueux emplissant l'espace," Acta Mathematica, vol. 63, no. 1, pp. 183-248, 1934.

[10] E. Hopf, "Über die Anfangswertaufgabe für die hydrodynamischen Grundgleichungen," Mathematische Nachrichten, vol. 4, pp. 213-231, 1951.

[11] D. Chae, "On the regularity conditions for the Navier-Stokes and related equations," Revista Mathemática Iberoamericana, vol. 23, no. 1, pp. 371-384, 2007.

[12] C. H. Chan and A. Vasseur, "Log improvement of the Prodi-Serrin criteria for Navier-Stokes equations," Methods and Applications of Analysis, vol. 14, no. 2, pp. 197-212, 2007.

[13] Z.-M. Chen and W. G. Price, "Morrey space techniques applied to the interior regularity problem of the Navier-Stokes equations," Nonlinearity, vol. 14, no. 6, pp. 1453-1472, 2001.

[14] J. Fan and T. Ozawa, "Regularity criterion for weak solutions to the Navier-Stokes equations in terms of the gradient of the pressure," Journal of Inequalities and Applications, Article ID 412678, 6 pages, 2008.

[15] J. Fan, S. Jiang, G. Nakamura, and Y. Zhou, "Logarithmically improved regularity criteria for the Navier-Stokes and MHD equations," Journal of Mathematical Fluid Mechanics, vol. 13, no. 4, pp. 557571, 2011.

[16] C. He, "New sufficient conditions for regularity of solutions to the Navier-Stokes equations," Advances in Mathematical Sciences and Applications, vol. 12, no. 2, pp. 535-548, 2002. 
[17] H. Kozono, T. Ogawa, and Y. Taniuchi, "The critical Sobolev inequalities in Besov spaces and regularity criterion to some semi-linear evolution equations," Mathematische Zeitschrift, vol. 242, no. 2, pp. 251-278, 2002.

[18] H. Kozono and N. Yatsu, "Extension criterion via two-components of vorticity on strong solutions to the 3D Navier-Stokes equations," Mathematische Zeitschrift, vol. 246, no. 1-2, pp. 55-68, 2004.

[19] I. Kukavica and M. Ziane, "One component regularity for the Navier-Stokes equations," Nonlinearity, vol. 19, no. 2, pp. 453-469, 2006.

[20] I. Kukavica and M. Ziane, "Navier-Stokes equations with regularity in one direction," Journal of Mathematical Physics, vol. 48, no. 6, pp. 065203-1-065203-10, 2007.

[21] I. Kukavica, "Regularity for the Navier-Stokes equations with a solution in a Morrey space," Indiana University Mathematics Journal, vol. 57, no. 6, pp. 2843-2860, 2008.

[22] J. Serrin, "On the interior regularity of weak solutions of the Navier-Stokes equations," Archive for Rational Mechanics and Analysis, vol. 9, pp. 187-195, 1962.

[23] Z. Zhang and Q. Chen, "Regularity criterion via two components of vorticity on weak solutions to the Navier-Stokes equations in $\mathbb{R}^{3}$," Journal of Differential Equations, vol. 216, no. 2, pp. 470-481, 2005.

[24] Y. Zhou and S. Gala, "Logarithmically improved regularity criteria for the Navier-Stokes equations in multiplier spaces," Journal of Mathematical Analysis and Applications, vol. 356, no. 2, pp. 498-501, 2009.

[25] Y. Zhou and M. Pokorný, "On a regularity criterion for the Navier-Stokes equations involving gradient of one velocity component," Journal of Mathematical Physics, vol. 50, no. 12, pp. 123514-1123514-11, 2009.

[26] Y. Zhou and M. Pokorný, “On the regularity of the solutions of the Navier-Stokes equations via one velocity component," Nonlinearity, vol. 23, no. 5, pp. 1097-1107, 2010.

[27] P. G. Lemarié-Rieusset, "The Navier-Stokes equations in the critical Morrey-Campanato space," Revista Mathemática Iberoamericana, vol. 23, no. 3, pp. 897-930, 2007.

[28] Y. Zhou and S. Gala, "A new regularity criterion for weak solutions to the viscous MHD equations in terms of the vorticity field," Nonlinear Analysis A, vol. 72, no. 9-10, pp. 3643-3648, 2010.

[29] T. Kato and G. Ponce, "Commutator estimates and the Euler and Navier-Stokes equations," Communications on Pure and Applied Mathematics, vol. 41, no. 7, pp. 891-907, 1988. 


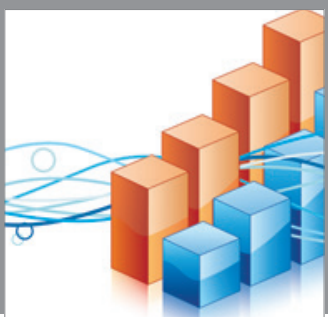

Advances in

Operations Research

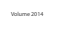

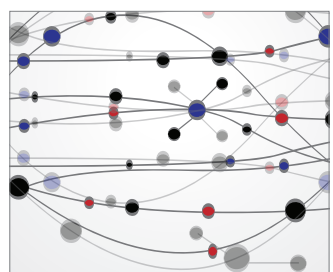

\section{The Scientific} World Journal
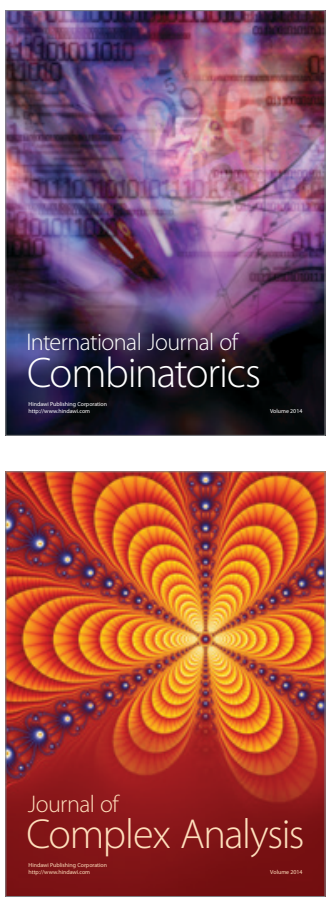

International Journal of

Mathematics and

Mathematical

Sciences
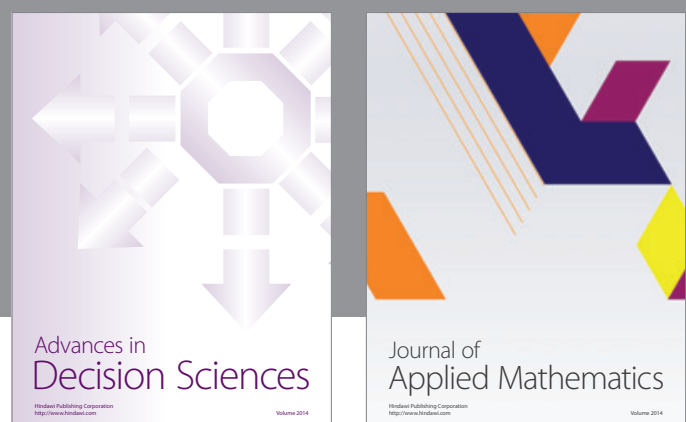

Journal of

Applied Mathematics
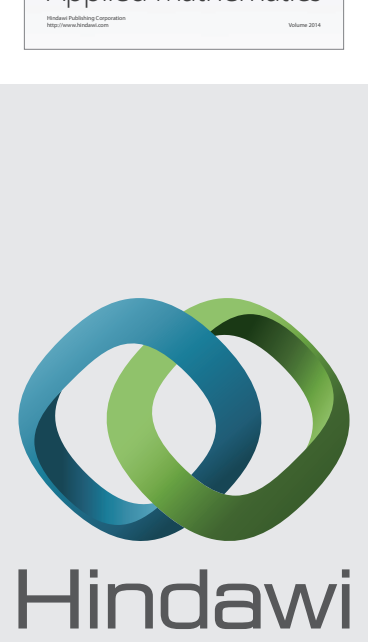

Submit your manuscripts at http://www.hindawi.com
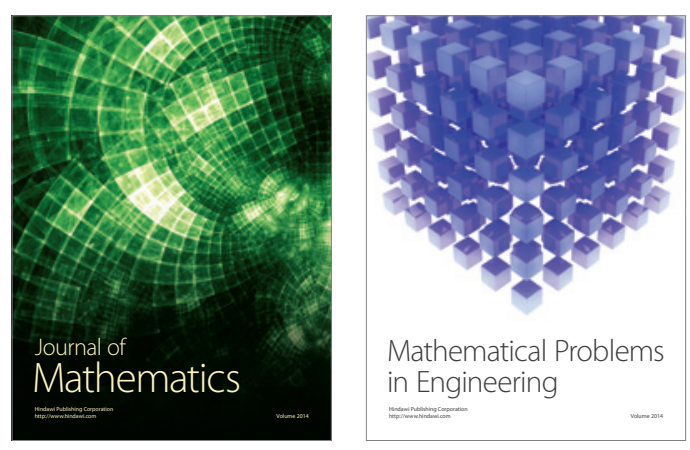

Mathematical Problems in Engineering
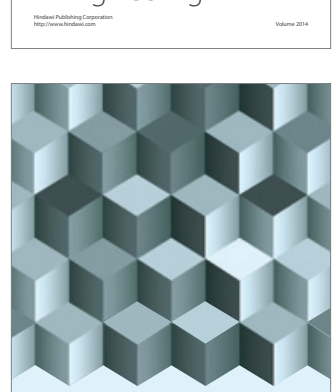

Journal of

Function Spaces
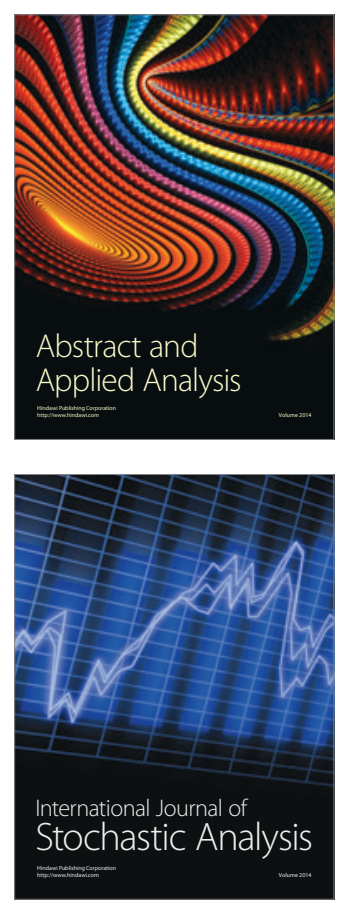

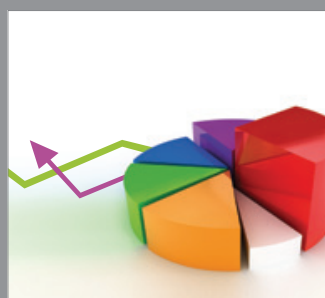

ournal of

Probability and Statistics

Promensencen
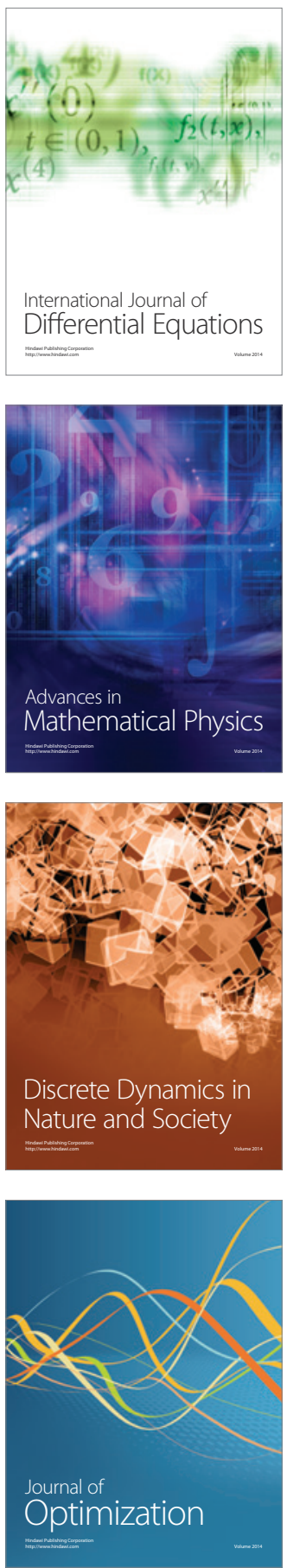